\title{
Adsorption of helium on a charged propeller molecule: hexaphenylbenzene
}

\author{
Siegfried Kollotzek ${ }^{1}$, Florent Calvo ${ }^{2}$, Serge Krasnokutski ${ }^{3}$, Fabio Zappa ${ }^{1}$, Paul Scheier ${ }^{1}$, and Olof Echt ${ }^{1,4, a}{ }^{(\mathbb{D}}$ \\ 1 Institut für Ionenphysik und Angewandte Physik, Universität Innsbruck, 6020 Innsbruck, Austria \\ 2 Université Grenoble Alpes, CNRS, LiPhy, 38000 Grenoble, France \\ 3 Laboratory Astrophysics and Cluster Physics Group of the MPI for Astronomy at the University of Jena, Helmholtzweg \\ 3, 07743 Jena, Germany \\ 4 Department of Physics, University of New Hampshire, Durham, NH 03824, USA
}

Received 16 September 2021 / Accepted 4 November 2021 / Published online 29 November 2021 (C) The Author(s) 2021

\begin{abstract}
Physisorption on planar or curved graphitic surfaces or aromatic rings has been investigated by various research groups, but in these studies, the substrate was usually strictly rigid. Here, we report a combined experimental and theoretical study of helium adsorption on cationic hexaphenylbenzene (HPB), a propeller-shaped molecule. The orientation of its propeller blades is known to be sensitive to the environment, with substantial differences between the molecule in the gas phase and in the crystalline solid. Mass spectra of $\mathrm{He}_{n} \mathrm{HPB}^{+}$, synthesized in helium nanodroplets, indicate enhanced stability for ions containing $n=2,4,14,28,42,44$, or 46 helium atoms. Path-integral molecular dynamics simulations reveal a significant dependence of the dissociation energy on the details of the HPB geometry. Good agreement between the experimental data and calculated dissociation energies is obtained, provided that the symmetry of $\mathrm{HPB}^{+}$is reduced from $D_{6}$ to $D_{2}$, such a lower symmetry being suggested from quantum chemical calculations as arising upon electron removal.
\end{abstract}

\section{Introduction}

The weak interaction of carbonaceous materials with helium and other atoms or molecules is of relevance to various scientific disciplines. Of special interest have been polycyclic aromatic hydrocarbons (PAHs) and allcarbon systems of finite size such as graphene flakes, nanotubes, and fullerenes. Fundamental phenomena such as structural isomerization, melting, orderdisorder, and wetting-nonwetting transitions have been explored by resonant two-photon ionization, fluorescence excitation, or emission spectroscopy of PAHs coated with noble gas atoms (for a review, see ref. [1]). A long-standing challenge in astrophysics, explaining the unidentified carriers of hundreds of diffuse absorption features of dense parts of the interstellar medium in the visible and near-infrared range, is possibly solved by PAHs, fullerenes, and other carbonaceous molecules [2]; inhomogeneous broadening due to adsorption of helium or hydrogen, the most abundant elements in the universe, may explain the unusually large width of the bands [3-7]. Likewise, the broad ultraviolet absorption bump observed at $217.5 \mathrm{~nm}$ in the interstellar medium extinction curve of galaxies may be due to hydrogenated

Supplementary Information The online version contains supplementary material available at https://doi.org/10.1140/ epjd/s10053-021-00301-6.

a e-mail: olof.echt@unh.edu (corresponding author)
PAHs or other carbonaceous materials [8]. Fullerenes and PAHs are also likely to play a role in astrochemistry [9].

Helium atoms are, among all adsorbates, most weakly bound; their polarizability is four times smaller than that of $\mathrm{H}_{2}[10,11]$. This raises challenges but also offers opportunities to the experimentalist and to the theoretician alike. A challenge, because weak binding requires low temperatures if the complex is to be formed in the gas phase. The calculated binding energy between, say, cationic fullerenes and helium is about 10 meV [12]; a simple rule-of-thumb predicts that helium would desorb from isolated $\mathrm{C}_{60}{ }^{+}$at $4 \mathrm{~K}$ within $\approx 10^{-6}$ $\mathrm{s}[13,14]$. Thus, forming long-lived helium- $\mathrm{C}_{60}+$ complexes in an ion trap requires temperatures well below $10 \mathrm{~K}[4]$.

Alternatively, one may start the synthesis of heliumion complexes by first forming neat helium nanodroplets (HNDs) in a supersonic expansion [15]. Their binding energies are close to the helium bulk value of 0.616 meV [16]; their spectroscopically measured temperature in vacuum is $0.37 \mathrm{~K}$ [17]. If these droplets collide with gas-phase ions, the ions will be picked up by the droplets, leading to the evaporation of enormous numbers of helium atoms until the temperature drops to $\approx 4 \mathrm{~K}$ or below, depending on the number of helium atoms that remain bound during the observational time window. An even better way to create ions with a small number of bound He atoms is to ion- 
ize HNDs, and then, let them collide with the neutral molecules. After the pickup, the charge is transferred to the dopant molecule. This process is very exothermic, leading to dopant cations with some attached He atoms [7].

As for the opportunities, $\mathrm{He}_{n} \mathrm{X}^{+}$ions are excellent candidates for messenger-type spectroscopy, where the absorption of a photon is detected via the depletion of size $n$. The low temperature greatly reduces spectral complexity, and the weak interaction implies minimal perturbation. Recording spectra as a function of $n$ helps to extrapolate spectral features to $n=0$, i.e., those of the bare ion.

The combination of weak binding, low temperature, and the small atomic mass of helium has another important consequence for the properties of $\mathrm{He}_{n} \mathrm{X}^{+}$, namely the possible appearance of nuclear delocalization of helium and its superfluidity [18]. Neutral HNDs (composed of the ${ }^{4} \mathrm{He}$ isotope) at $0.37 \mathrm{~K}$ are superfluid [17] although the interaction between helium and a dopant is usually enhanced if the dopant is positively charged. Therefore, the first (or first few) layers surrounding the dopant may be liquid-like, or even solid-like, forming an entity frequently referred to as a snowball $[15,19,20]$.

For helium adsorbed on PAHs, fullerenes, graphene, nanotubes, or other corrugated surfaces, the coverage and the energy barriers between preferred adsorption sites (in the hollows of the carbon rings) play an important role, too. Large barriers (whose size depends, e.g., on the curvature of the surface) will lead to stronger nuclear localization. Ordered layers that are commensurate with the surface corrugation will tend to be more strongly localized than layers with vacancies. In contrast, the hypothetical $1 \times 1$ phase, where each carbon ring of the honeycomb lattice is decorated by one helium atom, is crowded if one considers extended planar systems like graphene. The $1 \times 1$ phase will readily form on curved (convex) surfaces such as fullerenes and corannulene, while graphene supports the $\sqrt{ } 3 \times \sqrt{ } 3$ phase where all second-nearest carbon rings are covered $[12,21-28]$.

The preferred structure of helium layers formed on planar, positively charged PAHs has been explored by experiment and theory for anthracene $\left(\mathrm{C}_{14} \mathrm{H}_{10}\right)$, phenanthrene $\left(\mathrm{C}_{14} \mathrm{H}_{10}\right)$, fluoranthene $\left(\mathrm{C}_{16} \mathrm{H}_{10}\right)$, pyrene $\left(\mathrm{C}_{16} \mathrm{H}_{10}\right)$, and coronene $\left(\mathrm{C}_{24} \mathrm{H}_{12}, \mathrm{Cor}\right)[25,26,28]$. One might surmise that in these systems the crowded $1 \times 1$ phase is stabilized relative to adsorption on extended graphitic surfaces because the helium atoms adsorbed on the outer rings could relax outwards. But neither experiment nor theory support this naïve view, with the exception of neutral coronene $\left(\mathrm{C}_{24} \mathrm{H}_{12}\right.$, symmetry $\left.D_{6 h}\right)$ for which a classical study suggests that $14 \mathrm{He}$ atoms, one for each hollow site, will form a stable system [29]. However, quantum effects will destabilize the system $[24,26,29]$, and experiments do not show any enhanced stability for $\mathrm{He}_{14} \mathrm{Cor}^{+}$nor, for that matter, for $\left(\mathrm{H}_{2}\right)_{14} \mathrm{Cor}^{+}[25,30]$. Furthermore, the naïve model neglects that the peripheral $\mathrm{HCCH}$-bonds of PAHs offer additional favorable sites for helium.
In the present work, we explore helium adsorption on cationic hexaphenylbenzene $\left(\mathrm{C}_{42} \mathrm{H}_{30}, \mathrm{HPB}\right)$ which also involves planar units but, in contrast to all previously investigated PAHs and fullerenes, does not feature any adjacent carbon rings. Instead, the six phenyl rings are separated from the central benzene ring by CC bonds and one may expect that all 14 hollows (7 on each side) will easily accommodate one helium each. HPB, however, offers a complication: The six phenyl rings are tilted out of the plane because of steric hindrance. Electron diffraction data of (neutral) HPB in the gas phase reveal that the rings are nearly perpendicular to the central ring although they may oscillate with no appreciable restriction by approximately $\pm 10^{\circ}$ [31]. In the molecular crystal, the rings are rotated by about $65^{\circ}$ relative to the planar configuration, resulting in a propeller-like structure [32]. How will the propeller blades orient upon adsorption of helium? Moreover, is the structure altered by ionization, as required by mass spectrometry measurements?

In this work, we will present mass spectra of heliumHPB complexes. They are recorded by first forming helium nanodroplets in a supersonic expansion, ionizing the droplets by collisions with energetic electrons, passing the mass-to-charge selected HNDs through dilute HPB vapor leading to capture of HPB molecules in the droplets, gently stripping excess helium from the ions in a collision cell filled with helium gas at room temperature, and analyzing the size distribution of the emerging $\mathrm{He}_{n} \mathrm{HPB}^{+}$ions in a high-resolution time-offlight mass spectrometer. Local anomalies in the ion abundance versus size $n$ suggest enhanced stability of ions with $n=2,4,14,28,42,44$, and 46 adsorbed $\mathrm{He}$ atoms. To assist with the interpretation of these measurements, we have carried out some atomistic modeling of HPB coated with variable numbers $n=1-50$ of helium atoms, using the same computational methodology as in earlier works [23,24]. Three configurations were employed for HPB, namely two with symmetrically oriented phenyl rings as in the gas or crystalline phases, and one with a lower symmetry, as obtained from dedicated optimization by a quantum chemical method, in the cationic state. The results indicate a significant dependence of the dissociation energy on the details of the HPB geometry, those associated with the less symmetric configuration being in best agreement with the experiment.

\section{Methods}

\subsection{Experimental}

Small $\mathrm{He}_{n} \mathrm{HPB}^{+}$ions, with size $n$ typically ranging from 0 to about 100, are formed in and gently extracted from HNDs as follows: Neutral HNDs are grown by supersonic expansion of helium through a nozzle (diameter $5 \mu \mathrm{m}$, temperature $9.4 \mathrm{~K}$, stagnation pressure 22 bar) into ultra-high vacuum. The expanding beam is skimmed and ionized by electrons (energy $66 \mathrm{eV}$, cur- 
rent $350 \mu \mathrm{A}$ ). The resulting $\mathrm{He}_{N}{ }^{z+}$ ions are weakly accelerated into an electrostatic hemispherical deflector set to transmit HNDs with a size-to-charge ratio $N / z \approx 4.5 \times 10^{4}$. For simplicity, we discuss ions that are singly charged. The fate of multiply charged HNDs is discussed elsewhere [33,34].

The charged HNDs pass through a pickup cell into which vapor of HPB (obtained from Sigma-Aldrich, stated purity $98 \%$ ) is introduced from an external oven kept at $260^{\circ} \mathrm{C}$, and next an "evaporation cell" that contains helium at ambient temperature and low, variable pressure $P_{\mathrm{He}}$. Each collision will transfer, on average, $0.05 \mathrm{eV}$ to the HND, about 80 times the evaporation energy of a single helium atom from bulk helium [34]. Multiple collisions will lead to partial or complete evaporation of helium from the doped HND. The ions are guided by a radio-frequency field into the extraction region of a time-of-flight mass spectrometer (TOFMS) equipped with a reflectron in V-configuration. The mass resolution of the TOFMS is about 6000 (measured at full-width-at-half-maximum at mass $600 \mathrm{u}$ ). Additional experimental details have been published elsewhere [35].

\subsection{Computational}

$\mathrm{He}_{n} \mathrm{HPB}^{+}$clusters were modeled using a combination of classical global optimization techniques and pathintegral molecular dynamics simulations, following earlier works $[23,24]$. Briefly, we use a many-body force field to describe the interaction between the helium atoms and the $\mathrm{HPB}^{+}$ion, consisting of additive He$\mathrm{C}$, He-H repulsion-dispersion interaction and a polarizable contribution felt by each helium atom resulting from the distribution of partial charges on the hydrocarbon cation. The geometry of the $\mathrm{HPB}^{+}$ion is kept fixed throughout, assuming the phenyl rings to be all oriented perpendicularly to the central benzene ring, as in the gas phase for the neutral molecule ( $D_{6 h}$ point group), or with an angle of $65^{\circ}$ relative to the central ring, as in the crystal structure $\left(D_{6}\right.$ point group). Alternatively, local optimizations were carried out using density-functional theory at the $\mathrm{wB} 97 \mathrm{xD} / 6$ $31+\mathrm{G}^{*}$ level, resulting into a lower symmetry structure ( $D_{2}$ point group) with two opposite rings at $61^{\circ}$ and the other four at $53^{\circ}$ only. Such a symmetry breaking originates from a Jahn-Teller distortion upon ionizing the HPB molecule. For all three configurations, the partial charges on carbon and hydrogen atoms were determined using single point DFT/wB97xD/6-31+G* calculations and the standard RESP procedure [36]. The three geometries thus obtained are provided as supplementary material. All quantum chemical calculations were performed using the Gaussian09 software package [37].

For a given configuration of the $\mathrm{HPB}^{+}$ion, global optimization by basin-hopping [38] was first performed to identify low-energy structural candidates for 1 to 50 adsorbed helium atoms. For each cluster size, five independent series of $10^{5}$ local optimizations were carried

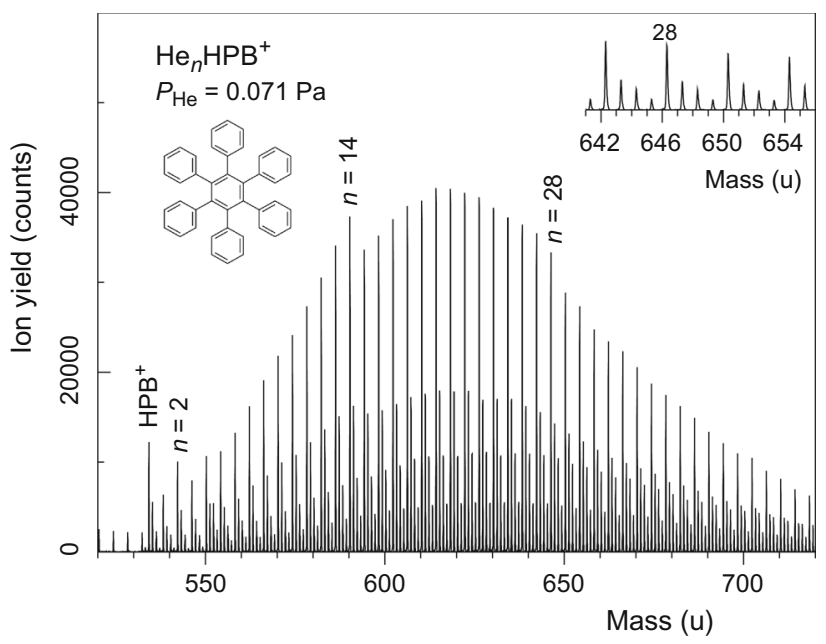

Fig. 1 Mass spectrum of helium nanodroplets (HNDs) doped with hexaphenylbenzene $\left(\mathrm{C}_{42} \mathrm{H}_{30}, \mathrm{HPB}\right)$. The most prominent mass peaks are due to isotopically pure $\mathrm{He}_{n}{ }^{12} \mathrm{C}_{42} \mathrm{H}_{30}{ }^{+}$; satellite peaks (see the inset) contain one or more ${ }^{13} \mathrm{C}$. Prominent local anomalies in the ion yield of $\mathrm{He}_{n} \mathrm{HPB}^{+}$versus size $n$ are indicated

out and a fictitious temperature of $10 \mathrm{~K}$ was employed to evaluate the Metropolis acceptance probabilities. Nuclear quantum effects were subsequently included by performing path-integral molecular dynamics (PIMD) simulations and evaluating the virial energy of the system, as well as various structural quantities. The PIMD trajectories were carried out at the temperature of $1 \mathrm{~K}$ and employed a Trotter discretization of 128, a time step of $0.5 \mathrm{fs}$. They were integrated over $1 \mathrm{~ns}$, averages being accumulated after 200 ps. Zero-point energy corrections to the static energies of the global minima were also determined in the harmonic approximation, but turned out to be particularly inaccurate to be considered as reliable. They are shown as additional information in the supplementary material.

\subsection{Results and discussion}

A mass spectrum of HNDs doped with HPB is displayed in Fig. 1. The pressure in the evaporation cell was set to $P_{\mathrm{He}}=0.071 \mathrm{~Pa}$. The series of prominent mass peaks, spaced at $4 \mathrm{u}$, is due to $\mathrm{He}_{n}{ }^{12} \mathrm{C}_{42} \mathrm{H}_{30}{ }^{+}$, $n \geq 0$. The inset in Fig. 1 reveals weaker satellite peaks that are due to $\mathrm{He}_{n} \mathrm{HPB}^{+}$containing one or more ${ }^{13} \mathrm{C}$, plus a weak contribution from ions contaminated by $\mathrm{H}_{2} \mathrm{O}$. Intramolecular fragments of $\mathrm{HPB}^{+}$are barely detectable. Their yield is much smaller than in the NIST mass spectrum recorded by electron ionization of bare HPB, in which fragment ions reach a maximum yield of $4.3 \%$ at mass $458 \mathrm{u}$ relative to the parent ion at $534 \mathrm{u}[39]$.

The envelope of the mass spectrum in Fig. 1 is quite smooth but small, statistically significant local anomalies appear at $n=2,14$, and 28. These anomalies can be identified more reliably when the mass spectrum is processed with the software IsotopeFit which accounts 


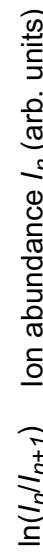
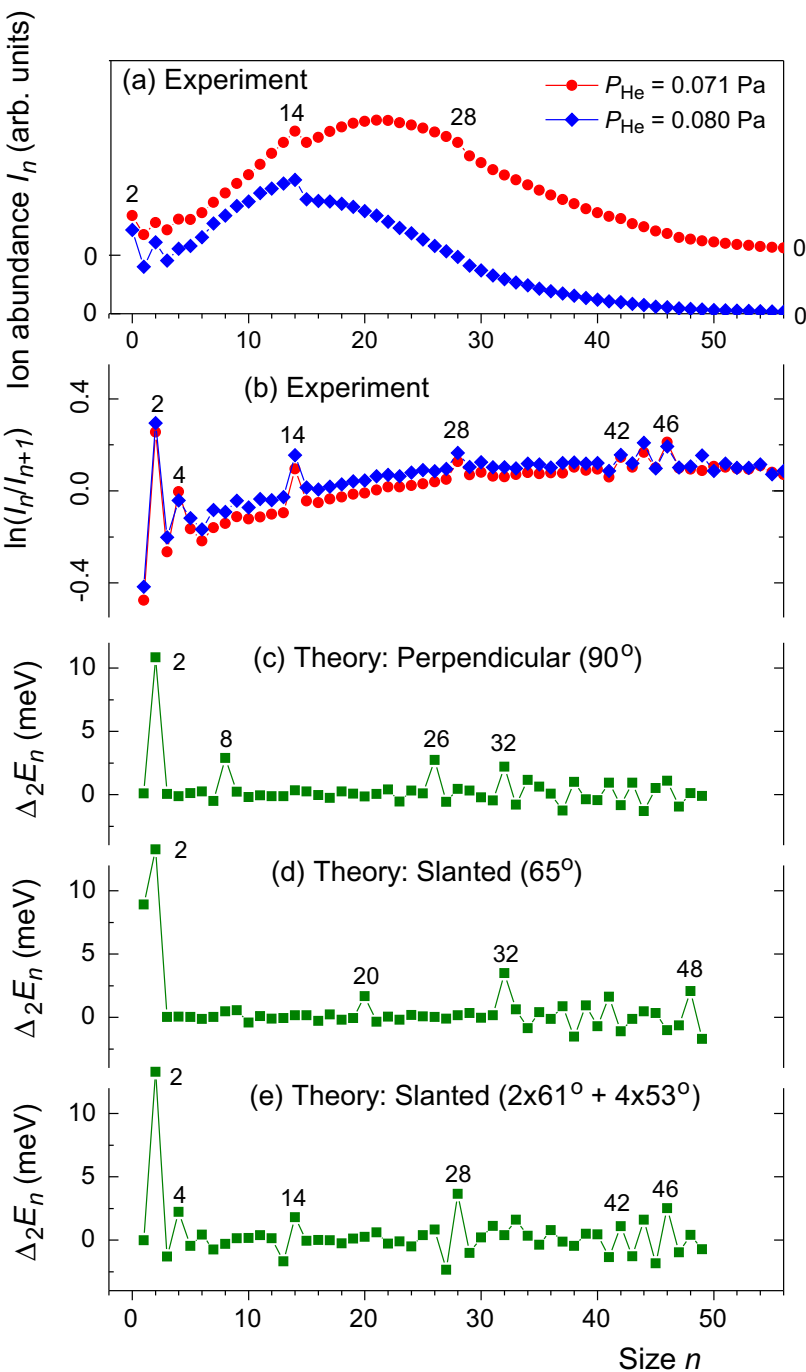

Fig. 2 a Ion abundance $I_{n}$ of $\mathrm{He}_{n} \mathrm{HPB}^{+}$versus size $n$ for two data sets recorded with different helium pressures in the evaporation cell. b First derivative of the logarithm of $I_{n}$. c-e Second derivate of the energies $E_{n}$ of $\mathrm{He}_{n} \mathrm{HPB}^{+}$calculated from the quantum virial energies for three different orientations of the phenyl groups. Prominent local anomalies are labeled

for isotopic patterns and assigns appropriate contributions of nominally isobaric ions [40]. The resulting ion abundance $I_{n}$ is plotted versus size $n$ in Fig. 2a. Two data sets are displayed; they are vertically stacked for better visibility. The one represented by red circles is extracted from the mass spectrum shown in Fig. 1. The other one (blue diamonds) is deduced from a spectrum that was recorded with a slightly higher evaporation pressure of $P_{\mathrm{He}}=0.080 \mathrm{~Pa}$. As a result, the abundance distribution is shifted to smaller values of $n$.

As the distribution shifts, some anomalies (e.g., at $n=14$ ) become more readily discernible while others (at $n=28$ ) are less easily spotted. Various procedures have been proposed for a remedy [20,41-43]. If, as in the present work, the observed cluster ions are the evap- orative products of larger clusters [13], the first derivative of the logarithmic ion abundance equals approximately the $2^{\text {nd }}$ derivative of the Helmholtz free energy $F_{n}$ divided by the Boltzmann constant $k_{\mathrm{B}}$ and vibrational temperature $T[42,44]$,

$$
-\Delta_{1} \ln I_{n}=\ln \frac{I_{n}}{I_{n+1}} \cong \Delta_{2} \frac{F_{n}}{k_{B} T}
$$

The concept of cluster temperature is subtle, and anomalies in the binding energy $E_{n}$ will be accompanied by anomalies in $T[13,45,46]$. On the other hand, HPB has many low-energy modes; hence, its heat capacity will remain relatively large even at very low temperatures [39], and the temperature decrease upon evaporation of a helium atom will be minor. If, furthermore, size dependencies of the entropy are neglected, one expects

$$
-\Delta_{1} \ln I_{n} \cong \text { const } \Delta_{2} E_{n}
$$

The graph of $-\Delta_{1} \ln I_{n}$ in Fig. 2b shows, indeed, pronounced local maxima at $n=2,14,28$ as well as several others, namely at $n=4$, and a triplet at 42,44 , 46. Additional anomalies (not shown in Fig. 2b) also appear at $n=57,59,66,68$, and 70 , but their statistical significance is not as clear.

The second derivatives obtained from the virial energies $E_{n}$ are displayed in Fig. 2 as a function of the number of helium atoms adsorbed on rigid $\mathrm{HPB}^{+}$ions assumed to have its propeller blades in a $D_{6 h}$ symmetric perpendicular, $D_{6}$ symmetric slanted, or $D_{2}$ asymmetric slanted configuration (panels c through e, respectively). In the three cases, the variations of the second energy derivative with increasing size are rather significant, and always exhibit a strong maximum at $n=$ 2. Except for this feature common to the three configurations, the remaining variations differ sensitively on the orientations of the propeller blades. Prominent sizes are thus found at $n=8,26$, and 32 for the symmetric perpendicular HPB cation, at $n=20$ and 32 for the symmetric slanted HPB cation, and at $n=4,14$, and 26 for the lower symmetry $D_{2}$ cation. Only the latter is compatible with our experimental measurements.

Before discussing the structural features inferred from these simulations, it is instructive to consider their classical limit and the energies associated with the classical global minima. The corresponding second derivatives are shown in Fig. $3 \mathrm{a}-\mathrm{c}$ for the three sets of clusters associated with the different geometries of the $\mathrm{HPB}^{+}$cation. Here, also the clusters with $n=2$ appear remarkably stable. However, the other prominent sizes usually do not match those obtained in the quantum calculations, nor do they resemble the experimental ion abundances either. Such differences are not really surprising, and were noticed earlier in similar systems [24]. They reflect the finite width of the nuclear wavefunction of helium atoms and the different ways these atoms accommodate with the various adsorption sites of the complex hydrocarbon molecule. These dif- 

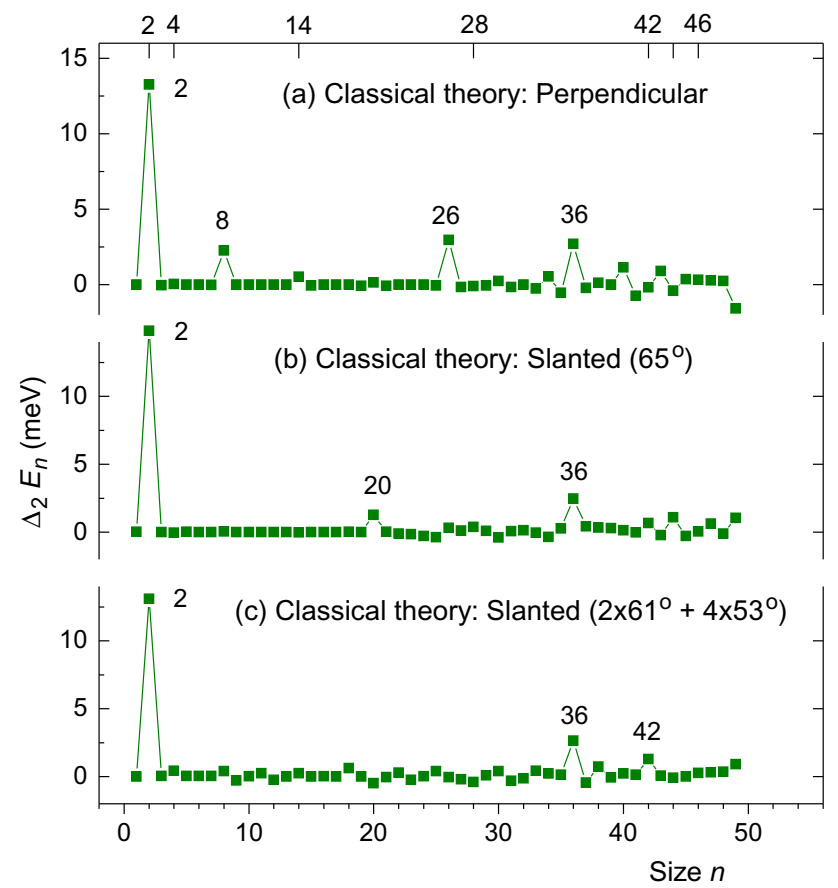

Fig. 3 Second derivative of the energies $E_{n}$ of $\mathrm{He}_{n} \mathrm{HPB}^{+}$ calculated from the classical global minima for three different orientations of the phenyl groups. Prominent local anomalies are labeled. Experimentally observed anomalies are indicated along the top ordinate

ferences originate from zero-point energy shifts but also sometimes from qualitatively different arrangements of the helium atoms. The classical structures (see supplementary material) exhibit such qualitative differences already at size $n=4$ for the low-symmetry HPB ion, where two atoms arrange on both sides of the central aromatic plane, whereas only two atoms are thus distributed for the two other HPB geometries, the remaining two atoms lying between successive propeller blades. That two atoms manage to be distributed on both sides of the central aromatic plane in the case of the slanted $D_{2}$ HPB ion with $n=4$ only originates from the slightly larger room available for this cation geometry, concomitantly with the generally lower angles exhibited by the lateral aromatic planes.

The quantum structures shown in Fig. 4 illustrate how the orientation of the propeller blades influences the preferred adsorption sites. The highly stable clusters found for $n=2$ correspond to the adsorption of one atom on each side of the central aromatic plane, which appears as the strongest binding site. We will refer to these two atoms as the central ones. Additional atoms favor the void regions between the propeller blades, but the symmetry of the HPB ion already affects the preferred regions to be filled. For the most symmetric $\left(D_{6 h}\right)$ ions, neighboring regions are filled first in order to maximize the van der Waals interaction between such atoms. However, for the less symmetric $D_{2}$ ions, the helium atoms have slightly more room when separated by propeller blades with different angles, lead-

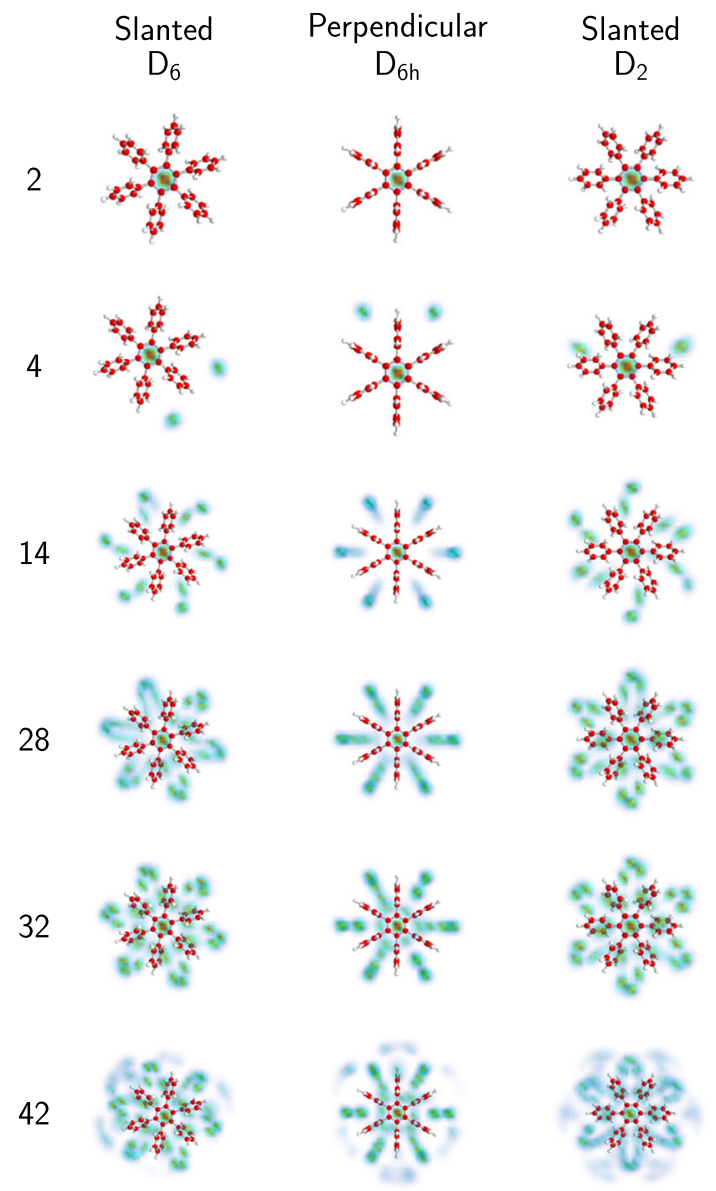

Fig. 4 Selected structures of $\mathrm{He}_{n} \mathrm{HPB}^{+}$clusters with $n=$ $2,4,14,28,32$, and 42 . For each size, the helium densities obtained from the PIMD simulations (shown in blue) are superimposed on the structure of HPB. In the $D_{2}$ slanted cation, the two propeller blades with the angle of $61^{\circ}$ are oriented toward the left and right, respectively

ing to the also rather stable structure at $n=4$. Above this size, the additional atoms have the choice of inserting themselves into another empty void between successive blades, or further filling existing regions. This competing process is not trivial, and also depends on the details of the HPB cation geometry. In particular, the growth is most symmetric for the $D_{6 h}$ perpendicular HPB ion, with the completion of a shell at $n=8$ with one atom in each void, in addition to the initial two atoms on both sides of the central aromatic ring, and a subsequent shell at $n=14$ with two atoms in each void, another one at $n=20$ with three atoms in each void, and yet another shell at $n=26$ with four atoms in each void, this size being associated with an enhanced binding energy. At $n=14$, the $D_{2}$ cation also binds helium atoms symmetrically as 2 atoms per void, although the preferred arrangement for 8 atoms is not so symmetric. At size 28, the favored structure for the helium atoms around the $\mathrm{HPB}^{+}$cation with $D_{2}$ symmetry has 2 central atoms, 3 atoms inside four of the voids, and 4 atoms in the remaining two voids that are 
symmetrically distributed between the propeller blades with $53^{\circ}$ tilting angle. The sixfold symmetric cations exhibit helium wavefunctions that are more delocalized, especially near the central two atoms.

At size $n=32$, the helium clusters are particularly stable when the $\mathrm{HPB}^{+}$cation is perpendicular $\left(D_{6 h}\right)$ or slanted $\left(D_{6}\right)$ symmetric, helium being distributed symmetrically as the two central atoms and 5 atoms in each of the six voids. This arrangement is also found around the $D_{2}$ cation, but does not appear to be energetically favorable relative to the neighboring sizes. Finally, the clusters at size 42 all display delocalized outer helium atoms, but the overall arrangement obtained from the PIMD simulations is much more regular for the $D_{2}$ cation than for the highly symmetric sixfold hydrocarbons. While the favored energetic stability of the 42-mer agrees with the prominent ion abundance in the experiment, the structural features are not so clear because, according to our simulations, this size lies beyond the completion of the first solvation shell. The clusters obtained at sizes 44 and 46 also display slightly higher stability when vibrational delocalization is accounted for and the HPB cation has $D_{2}$ symmetry, in agreement with the ion abundances. However, as in the case of $n=42$, the helium densities again show that the second solvation shell is partly filled at these sizes, no obvious symmetry being discernable in those densities. While the fluxional character of the second solvation shell has already been noticed in earlier investigations [6,20,23,26-29], we are thus cautious regarding the interpretation of the enhanced stabilities at sizes 42 and above.

Overall, our computational results reveal a significant dependence of the adsorption propensity of helium on the precise orientation of the propeller blades in the hexaphenylbenzene cation. The comparison between the measurements and the computational results obtained with three alternative geometries for the HPB cation indicate that only the low-symmetry $D_{2}$ configuration is compatible with the experimental data in the range covered in this study. While this obviously cannot rule out yet other configurations, sixfold symmetric, ideal geometries expected either in the gas phase or in the crystalline phase for the neutral HPB molecule, can be both excluded.

\section{Conclusion}

Adsorption of helium atoms on cationic hexaphenylbenzene was investigated by experimental mass spectrometry in helium nanodroplets and atomistic modeling using classical global optimization and quantum (pathintegral) molecular dynamics. The ion abundances show evidence for enhanced stabilities at specific sizes of 2, 4, 14, 28, 42, and 46 adsorbed atoms, such features being reproduced only if the hydrocarbon cation is assumed to be of lower symmetry ( $D_{2}$ point group) with respect to the sixfold symmetric point groups known to occur for the neutral molecule in both the crystal and gas phases.

We thus interpret the experimental ion abundances as providing indirect evidence for a symmetry breaking in the orientational distributions of the six propeller blades, which is predicted from quantum chemical calculations as a Jahn-Teller distortion upon ionizing the HPB molecule. Such effects are of a surprisingly large magnitude, as four of the propeller blades revert toward the angle of $53^{\circ}$.

Although our quantum modeling gives a fairly satisfactory account of the experimental measurements, it still relies on several approximations that would deserve further investigation. The rigid treatment used for the HPB cation is of particular interest, since the orientational degrees of freedom of the blades could be strongly prone to vibrational delocalization themselves. Moreover, the interplay between the flexibility of the cation and the presence of helium atoms should also become significant at higher helium loads. In principle, accounting for a flexible HPB cation in the presence of surrounding helium atoms in a quantum mechanical framework would be possible using simple but realistic force fields for the hydrocarbon cation, such as a tight-binding model with extra repulsion-dispersion terms [47]; however, we anticipate that some significant reparametrization would be needed to correctly reproduce the gas-phase quantum chemistry predictions.

Acknowledgements This work was supported by the Austrian Science Fund, FWF (Projects I4130, P31149, and W1259-N27). S.K. is grateful for the support by the Deutsche Forschungsgemeinschaft DFG (Grant KR 3995/4$1)$.

Funding Open access funding provided by University of Innsbruck and Medical University of Innsbruck.

Data Availability Statement This manuscript has data included as electronic supplementary material. The online version of this article contains supplementary material, which is available to authorized users.

Open Access This article is licensed under a Creative Commons Attribution 4.0 International License, which permits use, sharing, adaptation, distribution and reproduction in any medium or format, as long as you give appropriate credit to the original author(s) and the source, provide a link to the Creative Commons licence, and indicate if changes were made. The images or other third party material in this article are included in the article's Creative Commons licence, unless indicated otherwise in a credit line to the material. If material is not included in the article's Creative Commons licence and your intended use is not permitted by statutory regulation or exceeds the permitted use, you will need to obtain permission directly from the copyright holder. To view a copy of this licence, visit http://creativecomm ons.org/licenses/by/4.0/. 


\section{References}

1. S. Leutwyler, J. Bösiger, Rare-gas solvent clusters: spectra, structures, and order-disorder transitions. Chem. Rev. 90, 489-507 (1990). https://doi.org/10.1021/ cr00101a004

2. J. Fulara, J. Krelowski, Origin of diffuse interstellar bands: spectroscopic studies of their possible carriers. New Astron. Rev. 44, 581-597 (2000). https://doi.org/ 10.1016/S1387-6473(00)00108-1

3. C. Leidlmair, P. Bartl, H. Schöbel, S. Denifl, M. Probst, P. Scheier, O. Echt, On the possible presence of weakly bound fullerene- $\mathrm{H}_{2}$ complexes in the interstellar medium. Astrophys. J. Lett. 738, L4 (2011). https:// doi.org/10.1088/2041-8205/738/1/L4

4. E.K. Campbell, M. Holz, D. Gerlich, J.P. Maier, Laboratory confirmation of $\mathrm{C}_{60}{ }^{+}$as the carrier of two diffuse interstellar bands. Nature 523, 322-324 (2015). https:// doi.org/10.1038/nature14566

5. M. Kuhn et al., Atomically resolved phase transition of fullerene cations solvated in helium droplets. Nat. Commun. 7, 13550 (2016). https://doi.org/10.1038/ ncomms 13550

6. A. Kaiser et al., Isomeric broadening of $\mathrm{C}_{60}{ }^{+}$electronic excitation in helium droplets: experiments meet theory. J. Phys. Chem. Lett. 9, 1237-1242 (2018). https://doi. org/10.1021/acs.jpclett.8b00150

7. M. Meyer, P. Martini, A. Schiller, F. Zappa, S.A. Krasnokutski, P. Scheier, Electronic spectroscopy of anthracene cations and protonated anthracene in the search for carriers of diffuse interstellar bands. Astrophys. J. 913, 136 (2021). https://doi.org/10.3847/ 1538-4357/abf93e

8. C. Dubosq, F. Calvo, M. Rapacioli, E. Dartois, T. Pino, C. Falvo, A. Simon, Quantum modeling of the optical spectra of carbon cluster structural families and relation to the interstellar extinction UV bump. Astron. Astrophys. 634, A62 (2020). https://doi.org/10.1051/ 0004-6361/201937090

9. E. Herbst, The synthesis of large interstellar molecules. Int. Rev. Phys. Chem. 36, 287-331 (2017). https://doi. org/10.1080/0144235x.2017.1293974

10. J.K. Nagle, Atomic polarizability and electronegativity. J. Am. Chem. Soc. 112, 4741-4747 (1990). https://doi. org/10.1021/ja00168a019

11. T.N. Olney, N.M. Cann, G. Cooper, C.E. Brion, Absolute scale determination for photoabsorption spectra and the calculation of molecular properties using dipole sum rules. Chem. Phys. 223, 59-98 (1997). https://doi. org/10.1016/s0301-0104(97)00145-6

12. C. Leidlmair et al., Structures, energetics and dynamics of helium adsorbed on isolated fullerene ions. Phys. Rev. Lett. 108, 076101 (2012). https://doi.org/10.1103/ PhysRevLett.108.076101

13. C.E. Klots, Evaporation from small particles. J. Phys. Chem. 92, 5864-5868 (1988). https://doi.org/10.1021/ j100332a005

14. O. Echt, A. Kaiser, S. Zöttl, A. Mauracher, S. Denifl, P. Scheier, Adsorption of polar and non-polar molecules on isolated cationic $\mathrm{C}_{60}, \mathrm{C}_{70}$, and their aggregates. ChemPlusChem 78, 910-920 (2013). https://doi.org/ $10.1002 /$ cplu.201300198
15. A. Mauracher et al., Cold physics and chemistry: collisions, ionization and reactions inside helium nanodroplets close to zero K. Phys. Rep. 751, 1-90 (2018). https://doi.org/10.1016/j.physrep.2018.05.001

16. M. Rosenblit, J. Jortner, Electron bubbles in helium clusters. I. Structure and energetics. J. Chem. Phys. 124, 194505 (2006). https://doi.org/10.1063/1.2192780

17. J.P. Toennies, A.F. Vilesov, Superfluid helium droplets: a uniquely cold nanomatrix for molecules and molecular complexes. Angew. Chem., Int. Ed. 43, 2622-2648 (2004). https://doi.org/10.1002/anie.200300611

18. N. Issaoui, K. Abdessalem, H. Ghalla, S.J. Yaghmour, F. Calvo, B. Oujia, Theoretical investigation of the relative stability of $\mathrm{Na}^{+} \mathrm{He}_{n}(n=2-24)$ clusters: manybody versus delocalization effects. J. Chem. Phys. 141 , 174316 (2014). https://doi.org/10.1063/1.4900873

19. K.R. Atkins, Ions in liquid helium. Phys. Rev. 116, 1339-1343 (1959). https://doi.org/10.1103/PhysRev. 116.1339

20. T. González-Lezana, O. Echt, M. Gatchell, M. Bartolomei, J. Campos-Martínez, P. Scheier, Solvation of ions in helium. Int. Rev. Phys. Chem. 39, 465516 (2020). https://doi.org/10.1080/0144235X.2020. 1794585

21. D.S. Greywall, Heat capacity and the commensurateincommensurate transition of ${ }^{4} \mathrm{He}$ adsorbed on graphite. Phys. Rev. B 47, 309-318 (1993). https://doi.org/10. 1103/PhysRevB.47.309

22. H. Shin, Y. Kwon, Commensurate-incommensurate transition of ${ }^{4} \mathrm{He}$ adsorbed on a single $\mathrm{C}_{60}$ molecule. J. Chem. Phys. 136, 064514 (2012). https://doi.org/10. $1063 / 1.3685848$

23. F. Calvo, Size-induced melting and reentrant freezing in fullerene-doped helium clusters. Phys. Rev. B 85, 060502(R) (2012). https://doi.org/10.1103/PhysRevB. 85.060502

24. F. Calvo, Coating polycyclic aromatic hydrocarbon cations with helium clusters: snowballs and slush. J. Phys. Chem. A 119, 5959-5970 (2015). https://doi.org/ 10.1021/jp510799h

25. T. Kurzthaler, B. Rasul, M. Kuhn, A. Lindinger, P. Scheier, A.M. Ellis, The adsorption of helium atoms on coronene cations. J. Chem. Phys. 145, 064305 (2016). https://doi.org/10.1063/1.4960611

26. F. Calvo, Shell completion of helium atoms around the coronene cation. Comput. Theor. Chem. 1107, 2-6 (2017). https://doi.org/10.1016/j.comptc.2016.09.027

27. M. Gatchell, P. Martini, F. Laimer, M. Goulart, F. Calvo, P. Scheier, Spectroscopy of corannulene cations in helium nanodroplets. Faraday Discuss. 217, 276-289 (2019). https://doi.org/10.1039/c8fd00178b

28. A. Schiller, M. Meyer, P. Martini, F. Zappa, S. Krasnokutskiy, F. Calvo, P. Scheier, Adsorption of helium on small cationic PAHs: influence of hydrocarbon structure on the microsolvation pattern. J. Phys. Chem. A 125, 7813-7824 (2021). https://doi.org/10.1021/acs. jpca.1c05150

29. R. Rodriguez-Cantano et al., Coronene molecules in helium clusters: quantum and classical studies of energies and configurations. J. Chem. Phys. 143, 224306 (2015). https://doi.org/10.1063/1.4936414

30. M. Goulart, M. Kuhn, B. Rasul, J. Postler, M. Gatchell, H. Zettergren, P. Scheier, O. Echt, The structure of 
coronene cluster ions inferred from $\mathrm{H}_{2}$ uptake in the gas phase. Phys. Chem. Chem. Phys. 19, 27968-27973 (2017). https://doi.org/10.1039/C7CP04999D

31. A. Almenningen, O. Bastiansen, P.N. Skancke, Electron diffraction studies of hexaphenylbenzene vapour. Acta Chem. Scand. 12, 1215-1220 (1958). https://doi.org/ 10.3891 acta.chem.scand.12-1215

32. V. Vij, V. Bhalla, M. Kumar, Hexaarylbenzene: evolution of properties and applications of multitalented scaffold. Chem. Rev. 116, 9565-9627 (2016). https:// doi.org/10.1021/acs.chemrev.6b00144

33. L. Tiefenthaler, S. Kollotzek, A.M. Ellis, P. Scheier, O. Echt, Proton transfer at Subkelvin temperatures. Phys. Chem. Chem. Phys. 22, 28165-28172 (2020). https:// doi.org/10.1039/d0cp05174h

34. L. Tiefenthaler, S. Kollotzek, M. Gatchell, K. Hansen, P. Scheier, O. Echt, Isotope enrichment in neon clusters grown in helium nanodroplets. J. Chem. Phys. 153, 164305 (2020). https://doi.org/10.1063/5.0028056

35. L. Tiefenthaler et al., An intense source for cold cluster ions of a specific composition. Rev. Sci. Instrum. 91, 033315 (2020). https://doi.org/10.1063/1.5133112

36. J. Wang, K. Cieplak, K.A. Kollman, How well does a restrained electrostatic potential (RESP) model perform in calculating conformational energies of organic and biological molecules? J. Comput. Chem. 21, 1049-1074 (2000). https://doi.org/10.1002/ 1096-987X(200009)21:12〈1049::AID-JCC3 $>3.0 . C O ; 2-\mathrm{F}$

37. M.J. Frisch, G.W. Trucks, H.B. Schlegel, G.E. Scuseria, M.A. Robb, J.R. Cheeseman, G. Scalmani, V. Barone, B. Mennucci, G.A. Petersson et al. Gaussian 09, Revision E.01. Gaussian, Inc., Wallingford CT (2009)

38. D.J. Wales, J.P.K. Doye, Global optimization by basinhopping and the lowest energy structures of LennardJones clusters containing up to 110 atoms. J. Phys. Chem. A 101, 5111-5116 (1997). https://doi.org/10. 1021/jp970984n

39. Data from NIST Standard Reference Database 69: NIST Chemistry WebBook
40. S. Ralser, J. Postler, M. Harnisch, A.M. Ellis, P. Scheier, Extracting cluster distributions from mass spectra: isotopefit. Int. J. Mass Spectrom. 379, 194-199 (2015). https://doi.org/10.1016/j.ijms.2015.01.004

41. S. Prasalovich, K. Hansen, M. Kjellberg, V.N. Popok, E.E.B. Campbell, Surface entropy of rare-gas clusters. J. Chem. Phys. 123, 084317 (2005). https://doi.org/10. 1063/1.2008948

42. S. Bjørnholm, J. Borggreen, O. Echt, K. Hansen, J. Pedersen, H.D. Rasmussen, The influence of shells, electron thermodynamics, and evaporation on the abundance spectra of large sodium metal clusters. Z. Phys. D 19, 47-50 (1991). https://doi.org/10.1007/ 978-3-642-76178-2-9

43. W.A. De Heer, The physics of simple metal clusters: experimental aspects and simple models. Rev. Mod. Phys. 65, 611-676 (1993). https://doi.org/10. 1103/RevModPhys.65.611

44. M. Brack, O. Genzken, K. Hansen, Thermal electronic properties of alkali clusters. Z. Phys. D 19, 51-53 (1991). https://doi.org/10.1007/BF01448253

45. K. Hansen, U. Näher, Evaporation and cluster abundance spectra. Phys. Rev. A 60, 1240-1250 (1999). https://doi.org/10.1103/PhysRevA.60.1240

46. J.U. Andersen, E. Bonderup, K. Hansen, On the concept of temperature for a small isolated system. J. Chem. Phys. 114, 6518-6525 (2001). https://doi.org/10.1063/ 1.1357794

47. F. Calvo, C. Falvo, P. Parneix, A simple but accurate potential for the Naphthalene-Argon complex: applications to collisional energy transfer and matrix isolated IR spectroscopy. J. Chem. Phys. 138, 034305 (2013). https://doi.org/10.1063/1.4773469 